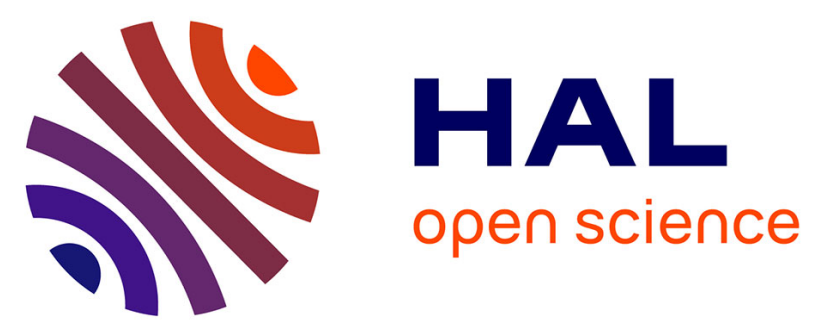

\title{
Electrodeless atmospheric secondary induced ionization jet (EASII-jet): Dynamics and properties of a transferred helium plasma source
}

Sylvain Iséni, Alibi Baitukha, Nelly Bonifaci, Cécile Pichard, Ahmed Khacef

\section{- To cite this version:}

Sylvain Iséni, Alibi Baitukha, Nelly Bonifaci, Cécile Pichard, Ahmed Khacef. Electrodeless atmospheric secondary induced ionization jet (EASII-jet): Dynamics and properties of a transferred helium plasma source. Physics of Plasmas, 2020, 27 (12), pp.123504. 10.1063/5.0027640 . hal-03064983

\section{HAL Id: hal-03064983 https://hal.science/hal-03064983}

Submitted on 14 Dec 2020

HAL is a multi-disciplinary open access archive for the deposit and dissemination of scientific research documents, whether they are published or not. The documents may come from teaching and research institutions in France or abroad, or from public or private research centers.
L'archive ouverte pluridisciplinaire $\mathbf{H A L}$, est destinée au dépôt et à la diffusion de documents scientifiques de niveau recherche, publiés ou non, émanant des établissements d'enseignement et de recherche français ou étrangers, des laboratoires publics ou privés. 


\title{
Electrodeless Atmospheric Secondary Induced Ionization jet: dynamics and properties of a transferred Helium plasma source
}

\author{
S. Iseni, ${ }^{1,}$ a) A. Baitukha, ${ }^{1}$ N. Bonifaci, ${ }^{2}$ C. Pichard, ${ }^{3}$ and A. Khacef ${ }^{1}$ \\ ${ }^{1)}$ GREMI (Groupe de Recherches sur l'Énergétique des Milieux Ionisés) - UMR7344 CNRS / Université d'Orléans, \\ 14 rue d'Issoudun, BP6744, 45067 Orléans, France. \\ ${ }^{2)}$ G2Elab (Laboratoire de Génie Électrique de Grenoble) - UMR5269 CNRS / \\ Grenoble INP / Université Grenoble Alpes, 21 rue des Martyrs, 38042 Grenoble, \\ France. \\ ${ }^{3)}$ Polytech Orléans, Université d'Orléans, Site Galilée, 12 rue de Blois, 45067 Orléans, \\ France.
}

(Dated: December 14, 2020)

EP - This paper was selected as an Editor's Pick.

This work introduces a versatile electrodeless cold plasma source in Helium based on an atmospheric pressure plasma jet (APPJ) device. The latter is used to generate guided ionization waves (IWs) impinging on a dielectric tube carrying He gas for the production of secondary induced ionization wave (SIIW). The dynamics of the plasma transfer across the dielectric is investigated by time resolved iCCD imaging technique and electrical diagnostics. Three operating modes are reported depending on the high voltage (HV) pulse duration which enables either a single or a double SIIW per HV pulse. Properties of the IWs and the double SIIW are reported. The gas temperature profiles of the plasma plumes expanding in to the ambient is investigated by optical emission spectroscopy. The results will be correlated to the time evolution of the current. This electrodeless atmospheric secondary induced ionization jet (EASII-jet) configuration can be easily implemented to electrically sensitive systems particularly in the fields of plasma medicine, pulsed electric field therapies and plasma activated liquids.

This is the Accepted Manuscript version -post-print- of an article accepted for publication in PHYSICS of Plasmas. This article may be downloaded for personal use only. Any other use requires prior permission of the author and AIP Publishing. This article appeared in Iseni S, Baitukha A, Bonifaci N, Pichard C, Khacef A. Electrodeless atmospheric secondary induced ionization jet (EASII-jet): Dynamics and properties of a transferred helium plasma source. Physics of Plasmas. 2020 Dec 1;27(12):123504, and may be found at doi:10.1063/5.0027640.

AIP Publishing is not responsible for any errors or omissions in this version of the manuscript or any version derived from it.

\section{INTRODUCTION}

For more than a decade, atmospheric pressure plasma jet (APPJ) devices ${ }^{1}$ have been under intensive research becoming a sizable part of the low temperature plasma community $^{2}$. The unique non-equilibrium plasma chemistry driven by energetic electrons -rather than thermal energy - is of significant interest to initiate chemical processes at moderate gas temperatures. Many applications rely on this plasma reactivity in catalysis and materials science processes ${ }^{3,4}$. More generally, APPJs are involved in a large panel of applications such as in medicine to stimulate biological responses ${ }^{5,6}$, agriculture ${ }^{7}$ and liquid activation $^{8,9}$. Beyond the non-equilibrium reactive chemistry, APPJs generate guided ionization waves ${ }^{10}$ (IWs) - guided streamers ${ }^{11}$ - driven by a high electric field (EF) ahead of the discharge channel ${ }^{12,13}$. After the breakdown in the vicinity of the powered electrode, the IWs propagate along the inner tube surface typically of several centimeters long and millimeters diameter before to expand

\footnotetext{
a)sylvain.iseni@univ-orleans.fr
}

in the ambient air. The dynamics of the IWs propagation is complex and depends on the hydrodynamics of the gas mixture, the geometry and the electrical excitation characteristics. Recent advances have shown remarkable controlling capabilities of the step-wise IW development by tailoring the applied voltage waveform enhancing the creation of a second ionization step ${ }^{13-15}$.

Following this intriguing approach, this work presents a variant configuration of APPJ, taking advantage of the intense EF to produce an electrodeless plasma source. In this research field, a couple of papers report on the observation of an IW re-ignition across dielectric tubes either with impinging on a metal electrode ${ }^{16}$ or directly through a Teflon pipe $^{17}$ while jet mode operation was not archived. The first success to stabilize a secondary jet dates back to the early time of guided streamers with the observation of a helium primary plasma which can propagate through the wall of a dielectric tube of constant diameter and keep propagating inside the dielectric tube secondary plasma ${ }^{18}$. The main mechanisms of IW plasma transfer behind a thin dielectric surface was thoroughly studied theoretically and numerically in a pin-to-plate air discharge configuration ${ }^{19,20}$ and in the case of an APPJs 
operating in Neon/Xenon mixture ${ }^{21}$. Although Helium gas is commonly used with APPJs, studies of electrodeless plasma source based on transferred IWs are hardly reported in He gas nor developed to a maturity stage. This work introduces an interesting design of electrodeless APPJ with the end active plasma region electrically isolated from the power supply. Taking advantage of the IW transfer across a dielectric, the dynamics and properties of the guided IWs differ from typical APPJ devices. One will report on different operating modes depending on the electrical properties of the excitation which is expected to significantly affect the reactive non-equilibrium chemistry.

\section{EXPERIMENTAL SETUP AND METHODS}

Conceptually, the EASII-jet configuration is made of two separated modules which are connected with the transient propagation of guided IWs. A schematic of the

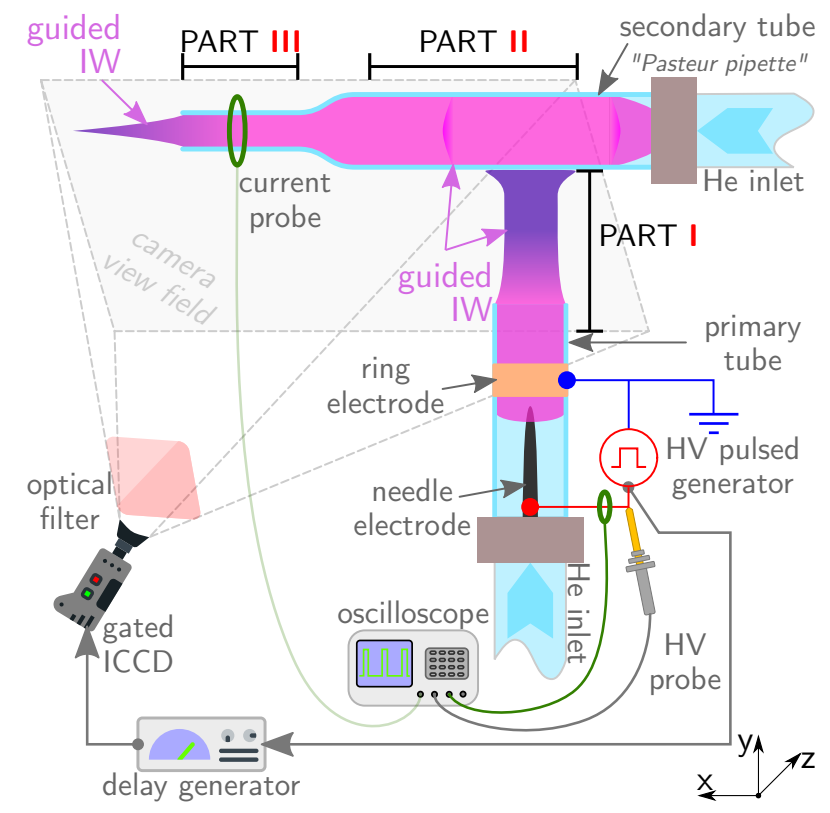

Figure 1. Schematics of the EASII-jet plasma source with the diagnostic systems. Three regions of interest are labeled as PART I, II and III.

plasma source configuration is shown in figure 1 . The first module consists of a typical APPJ in a dielectric barrier discharge (DBD)-like configuration ${ }^{1}$. Briefly, it is made of a Pyrex glass tube of $4.0 \mathrm{~mm}$ inner diameter and $100 \mathrm{~mm}$ long, equiped with a pair of electrodes. The powered tungsten needle electrode of $1.0 \mathrm{~mm}$ diameter has a tip radius of $(85 \pm 10) \mu \mathrm{m}$ and is centered within the primary tube. The grounded copper electrode is wrapped on the outer tube surface of $1 \mathrm{~mm}$ thickness. The APPJ is powered with a high voltage (HV) generator (nanogen3, RLC Electronic) delivering repetitive positive polarity pulses up to $15 \mathrm{kV}$ at frequencies up to $5 \mathrm{kHz}$. The pulse duration -or pulse width, $w_{\text {pulse }}{ }^{-}$ can be varied while keeping the amplitude constant with typical rise and fall times of $(98 \pm 4) \mathrm{ns}$. The characteristic output jitter time is bellow $0.1 \mathrm{~ns}$. The applied voltage is measured with a $\mathrm{HV}$ probe (model P6015A, Tektronix) and the total current with a current monitor sensor (model 4100, Pearson Electronics). To generate the guided IWs, He gas (Alphagaz 2, Air Liquide) flows inside the primary tube at a flow rate of 700 standard cubic centimeters per minute $(\mathrm{sccm})$ before to mix with the ambient air. This first module is set vertically with a He laminar flow directing upward in the opposite direction to the gravity force. This usual precaution enhances the stabilization of the IW propagation within the He plume expanding out of the primary tube and being subject to the buoyancy force ${ }^{22}$. After ignition of the discharge near the needle electrode, the IW is propagating within the primary tube ${ }^{23}$. At the outlet, the IW exits the tube and is guided by the He flow bounded by the surrounding air and flowing over several $\mathrm{cm}$ resembling a plasma plume $e^{23,24}$. This region of interest is labeled PART I in figure 1. This plasma plume -resulting from the propagation of successive guided $\mathrm{IWs}^{10}{ }^{10}$ connects the primary tube to the second module which consists of a Pasteur pipette carrying $300 \mathrm{sccm}$ of pure $\mathrm{He}$ gas. The Pasteur pipette is made of Pyrex glass (electric relative permittivity of $\left.\epsilon / \epsilon_{0}=4.7 \pm 0.6\right)^{25}$ and has a total length of $145 \mathrm{~mm}$. It is set at $5 \mathrm{~mm}$ away from the primary tube outlet and in the orthogonal direction with regards to the plasma plume expansion. Pipettes size 145 -ISO7712 certified- are used exclusively and the advantages are threefold: it is a standardized component (material and geometry), easily exchangeable, cheap and widely distributed to be commonly used in industry. The body -labeled PART II in figure 1- has a diameter of $(7.0 \pm 0.2) \mathrm{mm}$ and a length of $90 \mathrm{~mm}$. PART II ends with a constricted section of the body, narrowing to shape a long capillary of $(54 \pm 1) \mathrm{mm}$ long and labeled PART III in figure 1 . The capillary has an inner diameter of $(1.1 \pm 0.2) \mathrm{mm}$. A current monitor probe is also placed in PART III in order to measure the current of the secondary induced guided IWs. To hold both modules in place, a customized polymer frame was made with the help of a $3 \mathrm{D}$ printer.

It is worth noting that the Pasteur pipette is electrodeless. It is not electrically connected to the primary tube.Apart from being easily exchangeable, the main advantage of an electrodeless plasma source is to enhanced its compatibility with materials and devices vulnerable to current or voltage shock. For instance, this is an additional safety feature in plasma applied to biology and medicine with in vivo treatments ${ }^{26,27}$.

To image the propagation and the transfer of the discharge, an iCCD camera (model DH734, Andor Technology) with a $50 \mathrm{~mm}$ photographic lens is used. To resolve the propagation dynamics of the guided IWs, a delay generator synchronizes the iCCD gate with the HV generator. To better visualize the front of the IWs, an optical 
filter is used in order to isolate the He lines emission and to stop the second-positive-system of $\mathrm{N}_{2}$ diffusing from the ambient air into the plume.

The neutral gas temperature ( $\left.T_{\text {gas }}\right)$ is investigated in the primary and transferred IW by means of optical emission spectroscopy with the measurement of the rotational temperature $\left(T_{\text {rot }}\right)$ of $\mathrm{N}_{2}{ }^{28}$. This is a reliable method to obtain $T_{\text {gas }}$ in He discharges with $\mathrm{N}_{2}$ impurities ${ }^{29}$. Thus, one assumes $T_{\mathrm{g}} \approx T_{\text {rot }}$ and both will be used equivalently named in the following. Similar to reference ${ }^{22}$, the spectra are measured with a $0.75 \mathrm{~m}$ focal length imaging spectrograph (SP2750, Princeton Instruments) with a 1800 grooves $/ \mathrm{mm}$ diffraction grating. The spectra are recorded with a gated iCCD camera (PIMAX-III:1024i, $12.8 \mathrm{um}$ pixel size, Princeton Instruments) and triggered with the delay generator. The spectral resolution of the system is $(25 \pm 1) \mathrm{pm}$ at the band head $(380.5 \mathrm{~nm})$ of the $\mathrm{N}_{2}(\mathrm{C}-\mathrm{B})(0-2)$ vibrational band. The analysis of the rotational spectra is performed with a numerical tool developed in a previous study ${ }^{28}$. The parameters of the simulated spectrum are optimized with a non-linear leastsquare minimization algorithm to fit the experimental data. A particular attention is paid to the determination of the confidence interval of $T_{\text {rot }}$. This is realized by computing the $F$-test which is a robust approach in case of least squares optimization problems. Values of $T_{\text {rot }}$ are given with the confidence interval of $95.5 \%$.

\section{RESULTS AND DISCUSSION}

To investigate the dynamics of the EASII-jet plasma source, an iCCD image series of $0.8 \mu$ s total duration is presented in figure 2. The images are recorded after adjusting the trigger delay with regard to the rising edge of the HV pulses $((9.3 \pm 0.1) \mathrm{kV}$ amplitude at $5 \mathrm{kHz}$; $\left.w_{\text {pulse }}=2.0 \mu \mathrm{s}\right)$. In figure $\left.2 \mathrm{a}\right)$, the primary guided IW front is observed about $450 \mathrm{~ns}$ after the rising edge of the HV pulse. The luminous volume of light is characteristic of the IW front resulting from the space charge ahead of it, inducing an intense transient $\mathrm{EF}$ of values typically ranging from $10 \mathrm{kV} / \mathrm{cm}$ to $20 \mathrm{kV} / \mathrm{cm}$ in this type of discharge ${ }^{12,13,24,30}$. The propagation velocity of the guided IW is $(2.2 \pm 0.2) \times 10^{7} \mathrm{~cm} / \mathrm{s}$ in agreement with experimental and theoretical values reported in the literature $^{11,23,24,31}$. The total discharge current is recorded by placing the current monitor sensor around the anode electrode. Although the displacement current was not subtracted as suggested in $^{32}$, the current peak of the discharge up to $0.5 \mathrm{~A}$ is clearly identified on the voltage rising edge as shown in figure 3 for $w_{\text {pulse }}=10.0 \mu \mathrm{s}$. This peak value of the current amplitude is consistent with data from the literature ${ }^{18,33}$.

After exiting the primary tube, the IW continues its propagation in the ambient where it is guided by the molecular fraction of diffusing air into the He plume ${ }^{11,24}$. In figure 2b), the IW ahead of the conducting channel has reached the secondary tube outer surface and upon

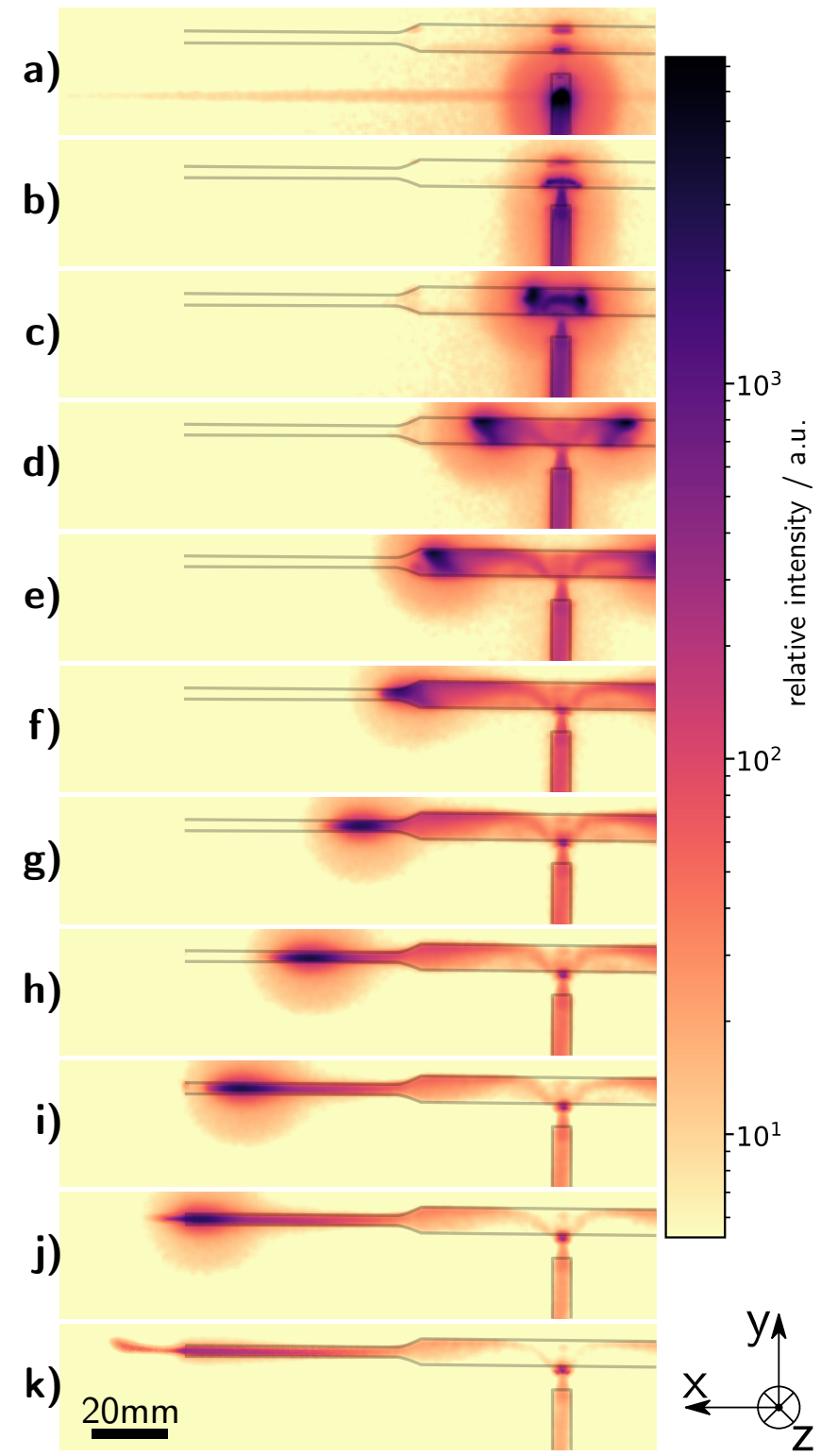

Figure 2. Sequence of successive iCCD images acquired after the $H V$ rising edge with $80 \mathrm{~ns}$ delay between each frame. The gate width is set to $5 \mathrm{~ns}$ and each frame results from an accumulation of $10^{4}$ triggered shots. The needle electrode is excited with $(9.3 \pm 0.1) \mathrm{kV}$ amplitude pulses at $5 \mathrm{kHz}$ with $w_{\text {pulse }}=2.0 \mu \mathrm{s}$.

impingement induces the transfer of the ionization within the electrodeless Pasteur pipette. The mechanisms governing this plasma transfer have been thoroughly studied theoretically and numerically for neon/xenon mixture ${ }^{21}$ and air ${ }^{19,20}$. Both models describe the same mechanisms for plasma transfer through a dielectric interface in positive polarity. Thus, it is expected to be of similar nature in case of noble gases such as He. After impinging on the outer glass surface, positive charges are being deposited resulting in a negative charging -mainly electrons- on the inner surface localized at the point of 
impact. The intense light emission at the inner surface -described here as a semi-ellipse area- results from the electron avalanche prior to the transferred IW development. Indeed, this luminous plasma volume is found to resemble the distribution of the ionization source where the electron density and electron temperature are particularly high ${ }^{21}$. This ionization source results mainly from the high EF at the primary guided IW front through the dielectric wall heating the seed electrons within the secondary tube. According to ${ }^{19-21}$, the surface charges deposited on the dielectric layer do not have a significant contribution to the ignition mechanism of the second IW in case of positive polarity. The photo-ionization is excluded to play a role in the transfer mechanism as Pyrex is not transparent to highly energetic photons.

In figure 2c) to e) two secondary induced IWs (SIIWs) propagate symmetrically from their ignition point in opposite directions. The IW fronts do not uniformly fill the volume of PART II and seem to develop onto the inner wall at a velocity of $(0.9 \pm 0.1) \times 10^{7} \mathrm{~cm} / \mathrm{s}$. Interestingly, the SIIWs propagate twice slower than the primary IWs. This could result from a lower electron density in PART II as well as lower electric potential upon the impingement than at the powered electrode ${ }^{21}$.

To make use of the SIIWs, the Pasteur pipette is a wise choice as it gains full advantage of the constricted section of PART II to PART III, narrowing the capillary section significantly. Consequently, the average gas velocity increases from $0.13 \mathrm{~m} / \mathrm{s}$ to $6.4 \mathrm{~m} / \mathrm{s}$ allowing for a longer He plume expanding into the ambient air. As shown in figure 2f) to i), the reduction of the inner diameter implies a transition mode of the SIIWs from wall hugging to a more homogeneous volume ionization front. One observes a small increase of the propagation velocity of $(1.3 \pm 0.1) \times 10^{7} \mathrm{~cm} / \mathrm{s}$ which is attributed to an enhancement of the EF due to an increase of the space charge width ahead of the IW front ${ }^{34,35}$. The SIIW continues its propagation beyond the capillary outlet to yield a plasma plume of (16 \pm 1$) \mathrm{mm}$ long as shown in figure $2 \mathrm{j}$ ) to $\mathrm{k}$ ). The asymmetry of the plasma plume is due to the buoyancy force acting on the He flow expanding into the ambient ${ }^{22}$ although IWs can induce hydrodynamic instabilities $^{36}$. Interestingly, the logarithmic color scale unveils the rather low light emission of the conducting channel left behind the SIIW path.

The current characteristics measured in PART III and presented in figure 3 reveal the propagation of the SIIWs with a delay compared to the primary IW. This delay of about $850 \mathrm{~ns}$ corresponds to the transfer and propagation time. As expected, the current peak of the SIIW produced by the rise of the HV potential is remarkably identical for different values of $w_{\text {pulse }}$ (see figure 3). Quantitatively, the current of the EASII-jet plasma plume is 50 times lower than the current of typical APPJs ${ }^{11,33,34}$. This is an interesting safety feature regarding the applications as the electric insulation relies on the dielectric material to prevent electric shock. For instance, this is particularly relevant in the field of plasma-medicine ${ }^{26,27}$.

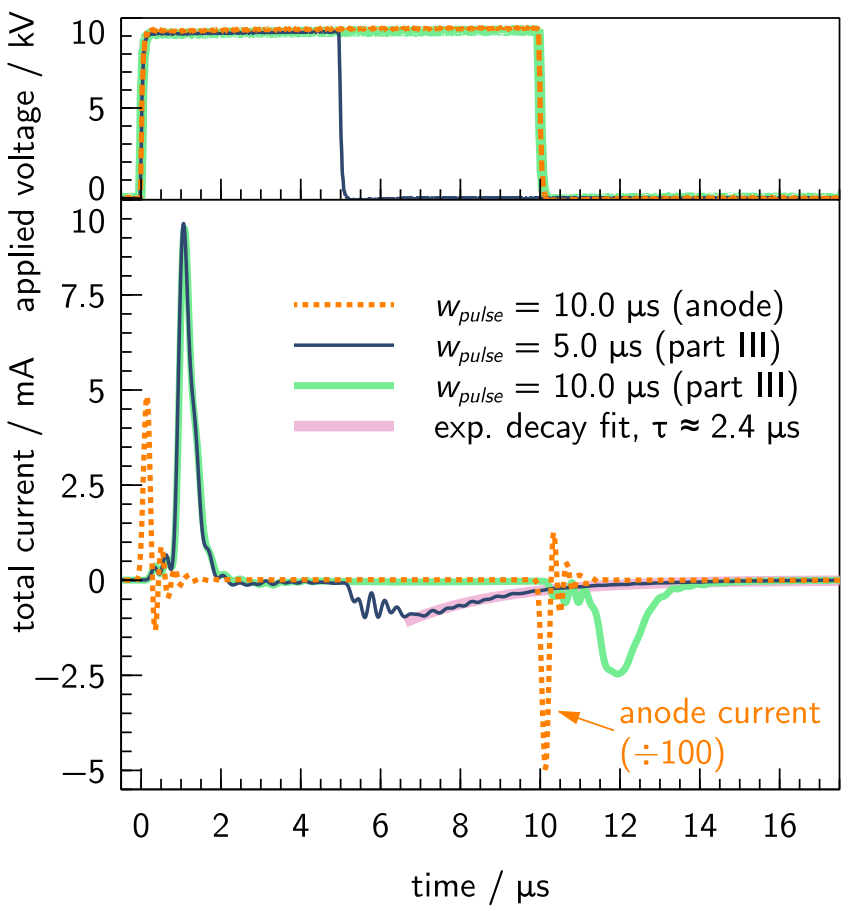

Figure 3. Time evolution of the applied voltage pulse and the corresponding total current measured on the EASII-jet plasma source for two values of pulse width, $w_{\text {pulse }}$, namely $5.0 \mu \mathrm{s}$ and $10.0 \mu \mathrm{s}$. The anode current curve is lowered by a factor 100 to scale with the $y$-axis.

Another aspect to consider -with the intrinsic nature of dielectric material interacting with ionized gas- is the role of the transient charging of the dielectric on the discharge dynamics and properties. Indeed, a recent study combining experimental and numerical investigations has brought substantial results on this concern ${ }^{37}$. The concluding remarks clarified the influence of the electrical excitation -namely polarity, HV amplitude and $w_{\text {pulse }}{ }^{-}$ on the charges deposited onto a dielectric surface. As the $\mathrm{HV}$ amplitude is known to affect the IW dynamics ${ }^{15}$, the $w_{\text {pulse }}$ was varied while keeping the HV amplitude fixed at $(9.3 \pm 0.1) \mathrm{kV}$ as reported in figure 3 . To support the electrical measurements, sequences of iCCD images are presented in figure 4 for three values of $w_{\text {pulse }}$. Under these experimental conditions, the charging of the Pasteur pipette wall is expected to vary with $w_{\text {pulse }}$. Therefore, one focuses now on the falling edge of the HV pulse. Figure 4-I) shows a series of the EASII-jet emission dynamics for $w_{\text {pulse }}=650 \mathrm{~ns}$. The rising edge of the HV pulse induces the propagation of the primary IW which produces in PART II the ignition of the SIIW (cf. figure 4-I)a) to d)). For very short $w_{\text {pulse }}$ values, the falling edge of the HV occurs during the SIIW is continuing its propagation. In the meantime the pulse falling edge triggers a new IW in the primary tube which impinges on the pipette outer wall. The potential drop of the electrode transferred by the plasma channel to the pipette correlates with the propagation stop of the SIIW within 


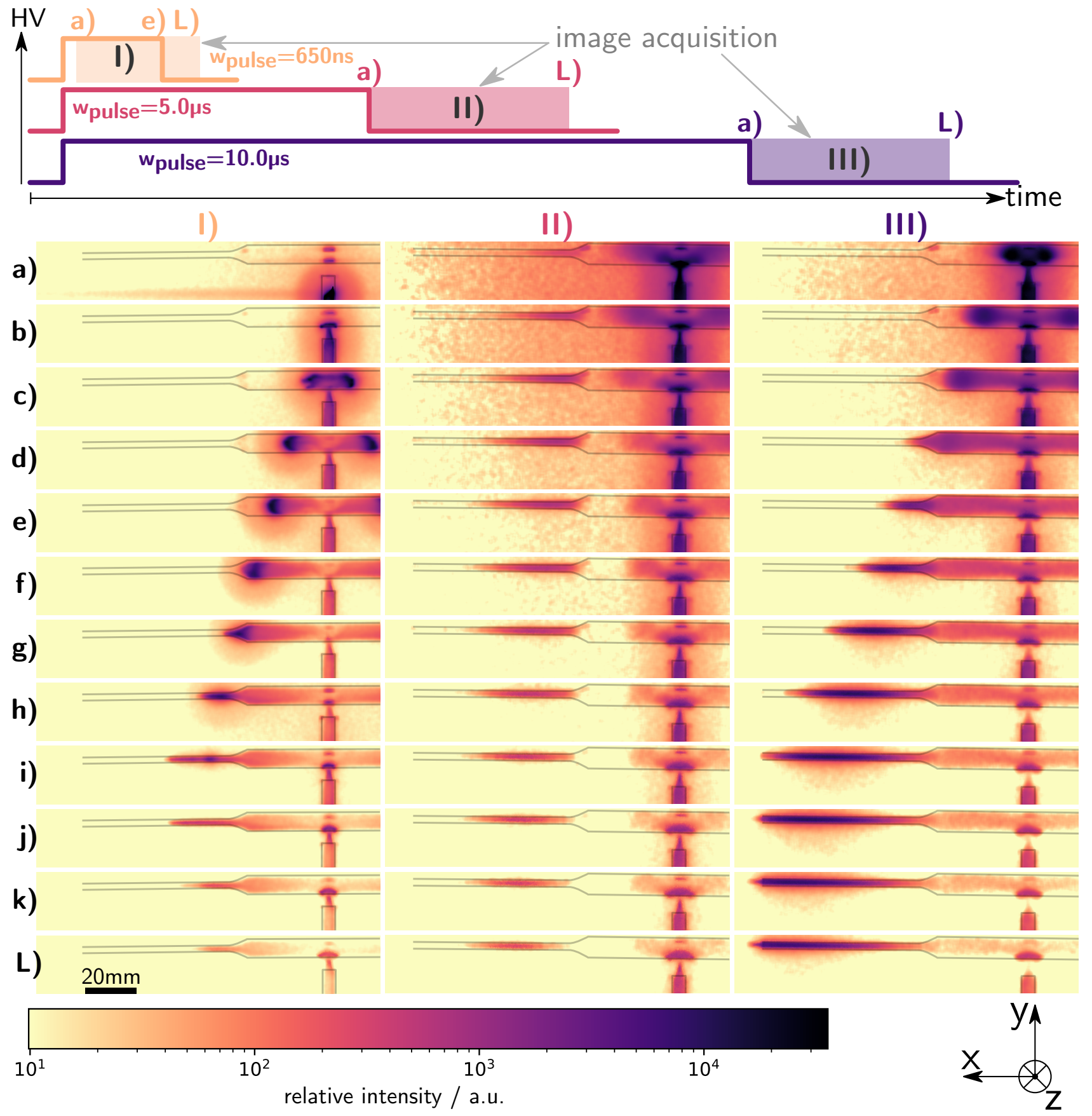

Figure 4. Sequence of successive iCCD images acquired for different values of $w_{\text {pulse }}$ with fixed electric excitation $((9.3 \pm 0.1) \mathrm{kV}$, $5 \mathrm{kHz}$ ). On top a timing diagram helps to identify the difference between the image series, particularly the image acquisition slots represented by the shaded area. Series I) corresponds to $w_{\text {pulse }}=650 \mathrm{~ns}$ with a delay between each frame of $80 \mathrm{~ns}$. Series II) and III) show the EASII-jet dynamics at the falling edge of the HV pulse for $w_{\text {pulse }}=5.0 \mu$ s and $w_{\text {pulse }}=10.0 \mu \mathrm{s}$ respectively, with $160 \mathrm{~ns}$ delay between each frame. (iCCD gate width of $5 \mathrm{~ns} ; 6 \times 10^{4}$ accumulated triggered shots.)

PART III (figure 4-I)i)). Interestingly the light emission from PART III suggests the persistence of a glow-like discharge. Similar observations are discussed in APPJs with small diameter capillary ${ }^{34}$. Interestingly, the bright semielliptical area emission at the point of impact is observed but does not develop further. Thus, there is no SIIW on the falling edge of the HV pulse in contrast to regular
APPJs $^{18,31,33,34}$.

Figure 4-II) and III) present the emission dynamics of the EASII-jet for larger values of $w_{\text {pulse }}$, namely $5.0 \mu \mathrm{s}$ and $10.0 \mu \mathrm{s}$ respectively. The corresponding applied voltage and current curves are presented in figure 3. For both series, images in row a) (cf. figure 4) captured the time of the plasma transfer is taking place, immediately after 
impingement of the primary IW. Intriguingly, a glowlike emission is observed at the constriction section of PART III for $w_{\text {pulse }}=5.0 \mu \mathrm{s}$ while for $w_{\text {pulse }}=10.0 \mu \mathrm{s}$, the Pasteur pipette does not emit any light. As the primary IW interacts with the outer surface, the semi-elliptical emission area at the inner surface -resembling the ionization source volume ${ }^{21}$ - tends to expand within a limited section of PART II as shown in series II) in figure 4. More surprisingly, the glow-like discharge within PART III is enhanced after plasma transfer but is clearly separated from the impingement zone of the primary IW in PART II. Indeed, the logarithmic color scale reveals the isolated discharge in PART III from which a current was recorded and shown in figure 3. Simultaneously with the falling edge of the HV pulse, the current curve for $w_{\text {pulse }}=5.0 \mu \mathrm{s}$ measured in PART III does not describe a typical waveform of IW. Similar to the emission, the current signal decays for several $\mu$ s before to return smoothly to zero. The monotonic decay rigorously fits with $I(t) \propto \exp \left(-\frac{t}{\tau}\right)$ by analogy with a discharging capacitor, with $\tau$ the decay time. The hypothesis of charges released from the wall to sustain the ionization is argued with the amount of charges deposited on dielectric surfaces as studied in ${ }^{37}$. The latter authors report on the crucial role of the HV amplitude and $w_{\text {pulse }}$ on the amount of deposited charges. In the present conditions, it is observed after the HV pulse returns to zero that the glow emission time is significantly longer for $w_{\text {pulse }}=5.0 \mathrm{\mu s}$ than for $w_{\text {pulse }}=650 \mathrm{~ns}$. This means that at constant voltage, the correlation between $w_{\text {pulse }}$ and the amount of deposited charges is consistent with the conclusions drawn in ${ }^{37}$.

Increasing values of $w_{\text {pulse }}$ from $10.0 \mu \mathrm{s}$ or larger will result in a different operating mode of the EASII-jet plasma source configuration. At first, the glow-like emission previously observed in PART III is not present at the falling edge of the HV as shown in figure 4-III)a). This is explained by the above mentioned argumentation of wall charging with a direct transfer of the HV potential from the electrode to the outer surface of the pipette and through the -partially- conductive channel within the primary tube. After impingement of the primary IW on the Pasteur pipette, the plasma transfer induces the ignition of a new SIIW. The latter propagates at the velocities of $(0.4 \pm 0.1) \times 10^{7} \mathrm{~cm} / \mathrm{s}$ and $(0.6 \pm 0.1) \times 10^{7} \mathrm{~cm} / \mathrm{s}$ in PART II and PART III respectively. The higher propagation velocity measured in PART III compared to the rising edge of the HV can be explained by a higher preionization from the previous decaying glow-like discharge. The related current evolution measured in PART III (figure 3) clearly reveals the current peak characteristic of the SIIW at $t=11.5 \mu \mathrm{s}$. The corresponding total current is found to be 200 times lower than for the primary IW which is electrically connected to the HV electrode. In these conditions, the SIIW yields a second plasma plume at the outlet at the pipette capillary pictured in figure 4III)j-L). One notes on this image series the existence of a continuous luminous conducting channel all along the Pasteur pipette left behind the path of the SIIW.
As a matter of interest, the transition mode from a single to a double SIIW per HV pulse was found around $w_{\text {pulse }}=(8 \pm 1) \mu \mathrm{s}$. Anecdotally, this could be acoustically estimated as the APPJ operates in the hearing range at a fixed frequency $(5 \mathrm{kHz})$ and the occurrence of the SIIW at the HV falling edge seems to change the acoustic which can be noticed by the human ear. This value of $w_{\text {pulse }}$ reduces with an increase of the $\mathrm{HV}$ amplitude. The latter parameter can be intricate to consider as it does change the IW propagation velocity while $w_{\text {pulse }}$ does not. However, this support the expected underlying mechanism to produce the double SIIW at the HV falling edge as it is expected to originate from the amount of charges accumulated onto the surface while maintaining the potential over $w_{\text {pulse }}=10.0 \mu \mathrm{s}$.

Considering the conditions to produce a double SIIW $\left(w_{\text {pulse }}=10.0 \mu \mathrm{s}\right)$, it is interesting to compare the gas temperature of the primary IW and the SIIW both propagating outside the tube. The axial profiles of $T_{\text {gas }}$ are measured along the propagation axis of each plasma plumes for the rising edge and the falling edge of the HV pulse. The results are shown in figure 5 . At the first glance values of $T_{\text {gas }}$ are found in a moderate range of temperatures which is consistent with typical values found in APPJs. Focusing on the rising edge of the HV (figure 5a)), one notices a clear difference between each profile with an average value $\left\langle T_{\text {gas }}\right\rangle$ around $360 \mathrm{~K}$ for the primary IW and $335 \mathrm{~K}$ for the SIIW. Although no temperature gradient is found in both cases with the first $4 \mathrm{~mm}, T_{\text {gas }}$ is measurably higher for the primary IW than in the SIIW. This is expected because of the energy is obviously coming from the primary IW before to be transferred. Taking in to account the confidence interval, $T_{\text {gas }}$ in the SIIW can be reasonably assumed as constant along all its propagation. This is not the case of the primary IW which exhibits of negative gradient from $4 \mathrm{~mm}$ to $5 \mathrm{~mm}$. This is explained with the plasma surface interaction with the Pasteur pipette outer all. The value of $T_{\text {gas }}$ measured in the vicinity of the surface is consistent with the data published in the literature ${ }^{38-40}$. Regarding $\left\langle T_{\text {gas }}\right\rangle$ in the plume of the SIIW, the latter is in agreement with prior measurements obtained in APPJ without interacting surface (free jet) ${ }^{22}$. Therefore, one expects that the kinetic of the long living reactive species would be governed similarly to common APPJ devices output.

The comparison with the IWs induced after the falling edge of the HV for $w_{\text {pulse }}=10.0 \mu \mathrm{s}$ now analyzed and discussed, particularly with regard to the double SIIW. The $T_{\text {gas }}$ profiles are presented in figure $5 \mathrm{~b}$ ). In the primary IW, $\left\langle T_{\text {gas }}\right\rangle$ seems slightly cooler but is clearly constant along the whole propagation until the surface of the Pasteur pipette. The absence of negative gradient in the vicinity of the wall can be explained with the moderate heating of the He channel during the pulse duration $(10.0 \mu \mathrm{s})$. This cannot be hypothesized in the case of two consecutive pulses delayed by $0.2 \mathrm{~ms}$ and where $T_{\text {gas }}$ is reasonably assumed to equal the ambient temperature. Regarding the SIIW produced at the HV falling edge, 

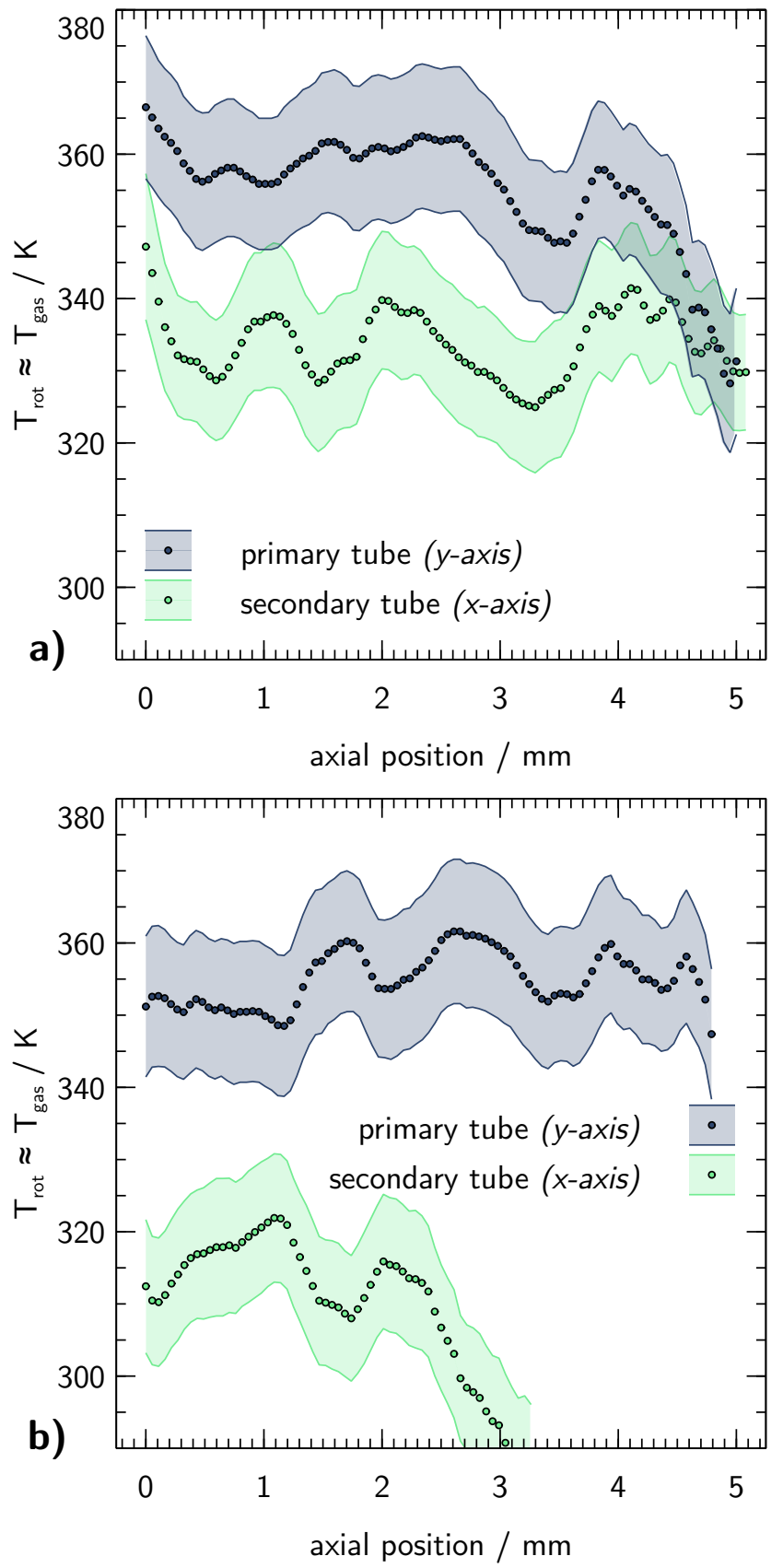

Figure 5. $T_{\text {rot }}$ of $\mathrm{N}_{2}(\mathrm{C}-\mathrm{B})(0-2)$ revealing the gas temperature profiles along the propagation axis of the primary IW (PART I) and the SIIW exiting the Paster pipette. Acquisition after the a) $H V$ rising edge and b) $H V$ falling edge, $10 \mu \mathrm{s}$ later. The position at $0 \mathrm{~mm}$ corresponds to the tube outlet. (iCCD gate width of $5 \mathrm{~ns} ; 10^{6}$ accumulated triggered shots; confidence interval of $95.5 \%$.)

the lower transferred energy has a direct effect on the propagation length. This was previously shown in figure 4-III)k-L). Similar to the current, $\left\langle T_{\text {gas }}\right\rangle$ is also lower by approximately $20 \mathrm{~K}$ compared to the SIIW induced after the rising edge of the HV. This quantitative temperature difference between the primary IW and the SIIW results from the dynamics of the discharge and supports the complementary properties of the initial APPJ concept.

\section{CONCLUDING REMARKS}

As a conclusion, the EASII-jet plasma source configuration offers attractive upgraded features to the conventional APPJ devices. Beyond the end-use plasma plume being electrically isolated from the power supply, EASII-jet configuration takes advantage of the plasma transfer mechanism across a dielectric surface ${ }^{20,21}$. Three operating modes are reported depending on the $w_{\text {pulse }}$ parameter. This enables to switch from a single to a double SIIWs generation per single HV pulse with a possible glow-like transition regime. The crucial role of the deposited charges on the dielectric surface is supported to explain the dynamics of the SIIW originating from the APPJ IW. In addition, the current peak of the SIIW is found to be from 50 to 200 lower than typical IWs current from APPJs. The temperature profile of the primary IW and of the double SIIW are clearly differentiated by approximately $30 \mathrm{~K}$. While the primary IW have the same value of $\left\langle T_{\text {gas }}\right\rangle$ for the discharge ignited after the rising edge and the following edge of the HV, this is not the case the for double SIIW. Indeed, the SIIW at the HV falling edge is weaker as well as a cooler $\left\langle T_{\text {gas }}\right\rangle$. The plasma chemistry -particularly the production of reactive species- is expected to differ from a typical APPJs which should open new perspectives of applications. For instance, the EASII-jet configuration offers the opportunity of local production of reactive species with intense pulsed EF desired to trigger biological mechanisms to support alternative cancer therapies ${ }^{6}$. The cheap and simple design of EASII-jet can also be implemented to integrated microfluidic systems to active liquids and surfaces ${ }^{9}$.

\section{DATA AVAILABILITY}

The data that support the findings of this study are available from the corresponding author upon reasonable request.

\section{REFERENCES}

${ }^{1}$ X. Lu, M. Laroussi, and V. Puech, Plasma Sources Science and Technology 21, 034005 (2012).

${ }^{2}$ I. Adamovich, S. D. Baalrud, A. Bogaerts, P. J. Bruggeman, M. Cappelli, V. Colombo, U. Czarnetzki, U. Ebert, J. G. Eden, P. Favia, D. B. Graves, S. Hamaguchi, G. Hieftje, M. Hori, I. D. Kaganovich, U. Kortshagen, M. J. Kushner, N. J. Mason, S. Mazouffre, S. M. Thagard, H.-R. Metelmann, A. Mizuno, E. Moreau, A. B. Murphy, B. A. Niemira, G. S. Oehrlein, Z. L. Petrovic, L. C. Pitchford, Y.-K. Pu, S. Rauf, O. Sakai, S. Samukawa, S. Starikovskaia, J. Tennyson, K Terashima, M. M. Turner, 
M. C. M. van de Sanden, and A. Vardelle, Journal of Physics D: Applied Physics 50, 323001 (2017).

${ }^{3}$ F. Massines, C. Sarra-Bournet, F. Fanelli, N. Naudé, and N. Gherardi, Plasma Processes and Polymers 9, 1041 (2012).

${ }^{4}$ A. Bogaerts, X. Tu, J. C. Whitehead, G. Centi, L. Lefferts, O. Guaitella, F. Azzolina-Jury, H.-H. Kim, A. B. Murphy, W. F. Schneider, T. Nozaki, J. C. Hicks, A. Rousseau, F. Thevenet, A. Khacef, and M. Carreon, Journal of Physics D: Applied Physics 53, 443001 (2020).

${ }^{5}$ J. Duan, L. Gan, L. Nie, F. Sun, X. Lu, and G. He, Physics of Plasmas 26, 043504 (2019).

${ }^{6}$ C. M. Wolff, A. Steuer, I. Stoffels, T. von Woedtke, K.-D. Weltmann, S. Bekeschus, and J. F. Kolb, Clinical Plasma Medicine 16, 100096 (2019)

${ }^{7}$ L. Sivachandiran and A. Khacef, RSC Advances 7, 1822 (2017). ${ }^{8}$ P. J. Bruggeman, M. J. Kushner, B. R. Locke, J. G. E. Gardeniers, W. G. Graham, D. B. Graves, R. C. H. M. Hofman-Caris, D Maric, J. P. Reid, E. Ceriani, D. F. Rivas, J. E. Foster, S. C. Garrick, Y. Gorbanev, S. Hamaguchi, F. Iza, H Jablonowski, E. Klimova, J. Kolb, F. Krcma, P. Lukes, Z. Machala, I. Marinov, D. Mariotti, S. M. Thagard, D Minakata, E. C. Neyts, J. Pawlat, Z. L. Petrovic, R. Pflieger, S. Reuter, D. C. Schram, S. Schröter, M. Shiraiwa, B Tarabová, P. A. Tsai, J. R. R. Verlet, T. von Woedtke, K. R. Wilson, K. Yasui, and G. Zvereva, Plasma Sources Science and Technology 25, 053002 (2016).

${ }^{9}$ O. Ogunyinka, A. Wright, G. Bolognesi, F. Iza, and H. C. H. Bandulasena, Microfluidics and Nanofluidics 24, 13 (2020).

${ }^{10} \mathrm{X}$. Lu and K. K. Ostrikov, Applied Physics Reviews 5, 031102 (2018).

${ }^{11}$ J.-P. Boeuf, L. L. Yang, and L. C. Pitchford, Journal of Physics D: Applied Physics 46, 015201 (2013).

${ }^{12}$ G. B. Sretenović, I. B. Krstić, V. V. Kovačević, B. M. Obradović, and M. M. Kuraica, Applied Physics Letters 99, 161502 (2011).

${ }^{13}$ G. V. Naidis and N. Y. Babaeva, Physics of Plasmas 26, 094501 (2019).

${ }^{14}$ M. E. Pinchuk, O. M. Stepanova, A. M. Astafiev, A. V. Lazukin, and Z. Chen, Applied Physics Letters 114, 194103 (2019).

${ }^{15}$ M. E. Pinchuk, O. M. Stepanova, M. Gromov, C. Leys, and A. Nikiforov, Applied Physics Letters 116, 164102 (2020)

${ }^{16} \mathrm{~S}$. Wu, Z. Wang, Q. Huang, Q. Xiong, and X. Lu, IEEE Transactions on Plasma Science 39, 2292 (2011).

${ }^{17}$ V. S. Johnson, W. Zhu, R. Wang, J. Lo Re, S. Sivaram, J. Mahoney, and J. L. Lopez, IEEE Transactions on Plasma Science 39, 2360 (2011).

${ }^{18}$ X. Lu, Q. Xiong, Z. Xiong, J. Hu, F. Zhou, W. Gong, Y. Xian, C. Zou, Z. Tang, Z. Jiang, and Y. Pan, Journal of Applied Physics 105, 043304 (2009).

${ }^{19} \mathrm{~F}$. Pechereau and A. Bourdon, Journal of Physics D: Applied Physics 47, 445206 (2014).

${ }^{20}$ F. Pechereau, J. Jánský, and A. Bourdon, Plasma Sources Science and Technology 21, 055011 (2012).
${ }^{21}$ Z. Xiong, E. Robert, V. Sarron, J.-M. Pouvesle, and M. J. Kushner, Journal of Physics D: Applied Physics 46, 155203 (2013).

${ }^{22}$ S. Iseni, C. Pichard, and A. Khacef, Applied Physics Letters 115, 034102 (2019).

${ }^{23}$ O. Stepanova, M. Pinchuk, A. Astafiev, and Z. Chen, Japanese Journal of Applied Physics 59, SHHC03 (2020).

${ }^{24}$ G. V. Naidis, Applied Physics Letters 98, 141501 (2011).

${ }^{25}$ H. P. R. Frederikse, in CRC Handbook of Chemistry and Physics: A Ready-Reference Book of Chemical and Physical Data., edited by W. M. Haynes, D. R. Lide, and T. J. Bruno (CRC Press, Boca Raton, USA, 2017) ninety-seventh ed., pp. 12-58.

${ }^{26}$ M. S. Mann, R. Tiede, K. Gavenis, G. Daeschlein, R. Bussiahn, K.-D. Weltmann, S. Emmert, T. von Woedtke, and R. Ahmed, Clinical Plasma Medicine 4, 35 (2016).

${ }^{27}$ H. Cheng, J. Xu, X. Li, D. Liu, and X. Lu, Physics of Plasmas 27, 063514 (2020).

${ }^{28}$ S. Iseni, Sylvain, R. Michaud, Ronan, P. Lefaucheux, G. B. Sretenović, V. Schulz-von der Gathen, and R. Dussart, Plasma Sources Science and Technology 28, 065003 (2019).

${ }^{29}$ P. J. Bruggeman, N. Sadeghi, D. C. Schram, and V. Linss, Plasma Sources Science and Technology 23, 023001 (2014).

${ }^{30}$ A. Sobota, O. Guaitella, G. B. Sretenović, V. V. Kovačević, E. Slikboer, I. B. Krstić, B. M. Obradović, and M. M. Kuraica, Plasma Sources Science and Technology 28, 045003 (2019).

${ }^{31}$ M. Hofmans, P. Viegas, O. van Rooij, B. Klarenaar, O. Guaitella, A. Bourdon, and A. Sobota, Plasma Sources Science and Technology 29, 034003 (2020).

${ }^{32}$ C. Douat, I. Kacem, N. Sadeghi, G. Bauville, M. Fleury, and V. Puech, Journal of Physics D: Applied Physics 49, 285204 (2016).

${ }^{33}$ E. R. W. V. Doremaele, V. S. S. K. Kondeti, and P. J. Bruggeman, Plasma Sources Science and Technology 27, 095006 (2018).

${ }^{34} \mathrm{~S}$. Wu, X. Lu, Y. Yue, X. Dong, and X. Pei, Physics of Plasmas 23, 103506 (2016).

${ }^{35}$ G. V. Naidis, Journal of Applied Physics 112, 103304 (2012).

${ }^{36}$ S. Iseni, A. Schmidt-Bleker, J. Winter, K.-D. Weltmann, and S. Reuter, Journal of Physics D: Applied Physics 47, 152001 (2014).

${ }^{37}$ E. Slikboer, P. Viegas, Z. Bonaventura, E. Garcia-Caurel, A. Sobota, A. Bourdon, and O. Guaitella, Plasma Sources Science and Technology 28, 095016 (2019).

${ }^{38}$ E. Slikboer, A. Sobota, O. Guaitella, and E. Garcia-Caurel, Journal of Physics D: Applied Physics 51, 025204 (2018).

${ }^{39}$ E. Slikboer, K. Acharya, A. Sobota, E. Garcia-Caurel, and O. Guaitella, Scientific Reports 10, 1 (2020).

${ }^{40}$ D. Gidon, D. B. Graves, and A. Mesbah, Plasma Sources Science and Technology 28, 025006 (2019). 